\title{
Implementation of an Evidence-Based Intervention with Safety Net Clinics to Improve Mammography Appointment Adherence Among Underserved Women
}

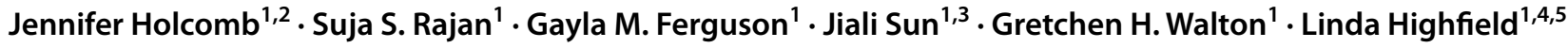

Accepted: 7 November 2021 / Published online: 25 November 2021

(c) The Author(s) 2021

\begin{abstract}
The Peace of Mind Program is an evidence-based intervention to improve mammography appointment adherence in underserved women. The aim of this study was to assess effectiveness of the intervention and implementation of the intervention in safety net clinics. The intervention was implemented through a non-randomized stepped wedge cluster hybrid study design with 19 Federally Qualified Health Centers and charity care clinics within the Greater Houston area. A multivariable generalized estimating equation logistic regression was conducted to examine mammography appointment adherence. A survey assessing Consolidated Framework for Implementation Research constructs was also conducted with clinic staff prior to adoption and eight weeks post implementation. One-sided $t$-tests were conducted to analyze mean score changes between the surveys. A total of 4402 women (baseline period $=2078$; intervention period $=2324$ ) were included in the final regression analysis. Women in the intervention period were more likely to attend or reschedule their mammography appointment $(\mathrm{OR}=1.30 ; p<0.01)$ than those in the baseline period receiving usual care. Women who completed the intervention were more likely to attend or reschedule their mammography appointment than those who did not complete the intervention $(\mathrm{OR}=1.62 ; p<0.01)$. The mammography appointment no-show rates for those in the baseline period, in the intervention period, and who completed the intervention were, respectively, 22\%, 19\%, and 15\%. A total of 15 clinics prior to adoption and eight clinics completed the survey at 8 weeks post implementation A statistically significant mean score decrease was observed in Inner Setting and in two Inner Setting CFIR constructs, Culture-Effort, and Implementation Climate. While the intervention improved mammography appointment adherence, there are opportunities to further integrate Consolidated Framework for Implementation Research constructs. Trial registration: Clinical trials registration number: NCT02296177.
\end{abstract}

Keywords Evidence-based intervention · Mammography adherence · Implementation science · Consolidated Framework for Implementation Research · Safety net clinics

Jennifer Holcomb

jennifer.1.holcomb@uth.tmc.edu

$\triangle$ Linda Highfield

linda.d.highfield@uth.tmc.edu

1 Department of Management, Policy and Community Health, School of Public Health, The University of Texas Health Science Center at Houston (UTHealth), 1200 Pressler St, Houston, TX 77030, USA

2 Sinai Urban Health Institute, 1500 South Fairfield Avenue, Chicago, IL 60608, USA
3 Houston Hospice, 1905 Holcombe Blvd, Houston, TX 77030, USA

4 Department of Epidemiology, Human Genetics and Environmental Sciences, School of Public Health, The University of Texas Health Science Center at Houston (UTHealth), 1200 Pressler St, Houston, TX 77030, USA

5 Department of Internal Medicine, The University of Texas Health Science Center at Houston (UTHealth) John P and Katherine G McGovern Medical School, 6431 Fannin St, Houston, TX 77030, USA 


\section{Background}

Breast cancer remains the second leading cause of cancer related death among women in the USA [1]. Mammography screening appointment adherence can be a critical component to receiving an early-stage breast cancer diagnosis [2]. Missing one mammography screening increases a woman's likelihood of developing a late-stage breast cancer [2-5]. Underserved women are at higher risk for late-stage diagnosis due to lower mammography screening rates, no-show appointments, and increased time between referral, diagnostic examination and treatment [6-10]. Understanding and addressing barriers to mammography appointment adherence in women who are not able to receive routine mammography screenings is the best way to reduce health disparities. Mammography screening interventions addressing both the structural (transportation and cost) and psychosocial (fear) barriers can promote appointment adherence in underserved communities [9, 10]. Patient navigation and counseling evidence-based interventions (EBIs) addressing these barriers have demonstrated success in increasing mammography appointment adherence [9-12]. Although mammography screening interventions might alter mammography screening behavior, to be successful, interventions should be adapted and tailored to meet community needs [10-12].

Dissemination and implementation (D\&I) practitioners aiming to implement a tailored intervention to intercede across cost and access to care among underserved communities have also partnered with safety net healthcare systems and Federally Qualified Health Centers (FQHCs). FQHCs provide culturally competent primary care and preventative services in underserved communities [10]. The nature of FQHCs lends them direct access to underserved women in need of mammography screening services, but also creates barriers to EBI adoption and implementation $[13,14]$. There is a gap in understanding of effective D\&I methods in successful interventions, particularly those aimed at addressing mammography appointment barriers within safety net clinics [15]. The current study utilized a theoretically based, D\&I strategy to facilitate the implementation of an EBI with FQHCs and charity care clinics and mammography mobile providers to improve mammography appointment adherence in underserved women. The Peace of Mind Program (PMP) intervention is an evidence-based mammography screening intervention based on the Transtheoretical Model of Change adapted from a research tested program from the National Cancer Institute's Research Tested Intervention Programs (RTIPs) database $[15,16]$. In this study, the PMP intervention was expanded to include implementation components geared toward supporting implementation in safety net clinics, and by doing so, aimed to address the research to practice gap $[15,16]$. We hypothesized that the intervention would improve mammography appointment adherence compared to usual care. The study objectives were to test the effectiveness of the intervention in improving mammography appointment adherence and to assess implementation of the intervention.

\section{Methods}

\section{Intervention}

PMP is an active listening, tailored telephone reminder call intervention to counsel women through barriers to mammography screening appointment attendance $[15,16]$. Each woman during the intervention period received up to three reminder call attempts for their scheduled mammography screening appointment at the safety net clinic. If the woman did not answer on the first attempt, two additional attempts were made to reach the woman. If the woman answered the phone call, but did not consent to participate in the study, she was reminded about her appointment in the usual care manner of each site. If she answered and consented to participate, a state certified Community Health Worker (CHW) who made the call assessed the woman's confidence in attending their scheduled mammography appointment, counseled the woman through barriers to attending the appointment (e.g., transportation, childcare), and recorded the woman's responses in an online interface program designed in RedCap. The results of each reminder phone call, whether the call was completed or a message left, and the woman's resulting mammography appointment attendance or no-show status were also recorded.

To adopt the intervention, clinics must have been members of the Breast Health Collaborative of Texas (BHCTexas) within the Greater Houston service area. BHCTexas is a statewide member network of breast cancer survivors, advocates, health care professionals, and organizations providing mammography and other breast health services.

BHCTexas and research team worked with participating clinics to align goals for mobile mammography drives and facilitated relationships with mobile providers as needed to support PMP implementation. Training support and onsite role modeling from BHCTexas CHWs was provided throughout implementation to clinic $\mathrm{CHWs}$ delivering the intervention. In addition, participating clinics must (1) have had a designation as a FQHC by the Health Resources and Services Administration (HRSA) or be a charity clinic which provides free or reduced cost care to underserved populations in their service area, (2) serve women between the ages of 40 to 64 years old who were at or below $200 \%$ of the Federal Poverty Level for a family of four and who lacked health 
insurance, (3) engage in provision of mammography screening services at least six times per year (three in baseline and in intervention), and (4) women at the clinic must have been in need of mammography screening and be scheduled for an upcoming appointment. Patients must have completed a clinical breast exam prior to their scheduled appointment per mobile provider requirements. A full description of the intervention development, adoption, implementation, and stakeholder engagement components has been reported elsewhere [15-17].

\section{Study Design}

A non-randomized stepped wedge cluster hybrid design was used to assign clinics into two non-concurrent implementation waves with two to three groups in each wave [15]. Variation across clinics existed in frequency of mammography drives, number of patients scheduled in each drive, number of staff available to participate in PMP, existing relationships with mammography mobile providers, and available funding and resources which resulted in differences in clinic readiness to start the intervention. Due to these differences, the randomized allocation of the clinics into waves and groups as previously described was not possible [15]. Clinics with lower levels of readiness were assigned to later groups to benefit from more time in the implementation strategies. Each clinic served as its own control during the baseline period and was required to have at least three mammography drives during both the baseline and intervention period. Since each clinic served as its own control, blinding was not possible.

To test effectiveness, the outcome measure was mammography appointment adherence. A patient's adherence was categorized as " 0 " for a no-show or cancelled appointment and categorized as " 1 " for an attended or rescheduled appointment. The independent variables of interest were two dichotomous variables indicating study period, baseline period (categorized as " 0 ") or intervention period (categorized as " 1 ") and for the intervention period, whether the patient did not complete (categorized as " 0 ") or did complete (categorized as "1") the intervention. A patient completed the intervention if they answered the reminder call, consented to the study, and received the staging question assessing confidence and barriers. Patient, intervention, clinic, and implementation covariates were also examined. Patient age was categorized into three age groups- -55 and above, 45 to 54 and 25 to 44 years - to align with the age-based mammography screening guidelines. The season in which the patient scheduled their appointment was categorized by winter (January to March), spring (April to June), summer (July to September), and fall (October to December) to examine a possible seasonal effect $[18,19]$. Wave was categorized as a dichotomous variable (0/1) for wave 1 and 2 and group was categorized as a three-category variable $(1,2,3)$ based on when the clinic began the intervention. Each of the three mammography mobile providers were categorized as a dichotomous variable based on if the provider assisted with mammography screenings at the clinic in which the patient was scheduled (categorized as " 1 ") or not scheduled (categorized as " 0 ") for a mammography appointment. The provider with an existing reminder call and group education program for usual care was defined as the reference group. The clinic racial/ethnicity distribution (percentage) of the population served by each clinic from 2015 to 2016 was collected from the HRSA Uniform Data System for FQHCs and the Texas Association of Community Health Centers for charity care clinics. The clinics were categorized based on the racial/ ethnicity group with the highest percentage in five mutually exclusive groups: non-Hispanic Black, non-Hispanic white, non-Hispanic other (another race other than Black or white), Hispanic, and multi-racial/ethnicity group (equal percentage of non-Hispanic Black, non-Hispanic other, and Hispanic women served). Each of the five groups were categorized as a dichotomous variable indicating if the racial/ethnicity group was the highest reported for the clinic (categorized as "1") or not (categorized as "0"). The CHW who made the reminder call was a dichotomous variable based on if they were a clinic (categorized as "0") or BHCTexas (categorized as " 1 ") staff member. A dichotomous variable determined if the patient answered the reminder call (categorized as " 1 ") or not (categorized as " 0 "). The number of reminder call attempts received by patient in the intervention period was a dichotomous variable for one call (categorized as " 0 ") or multiple call attempts (two or three calls categorized as "1"). Language was categorized by English, Spanish, or Vietnamese. To assess implementation, a survey was conducted with clinic leadership and staff with any potential role in PMP prior to adoption of the intervention and eight weeks into implementation to assess the same Consolidated Framework for Implementation Research (CFIR) constructs. A total of 75 survey statement items were used to assess twelve constructs across three CFIR domains using a survey adapted from the Cancer Prevention and Control Research Network for cancer control EBIs with FQHCs [Appendix] [20-22]. A mean score for each clinic was created to measure level of agreement with survey statement items $(5=$ completely agree to $1=$ completely disagree). Twenty survey statements were recoded to align with level of agreement and scoring direction (e.g., It will be hard to train providers and staff to implement the PMP).

\section{Data Analysis}

A descriptive analysis was performed to examine differences in covariates across the baseline and intervention periods. To test for statistically significant differences in mammography 
appointment adherence, we used chi-square tests. We used a multivariable generalized estimating equation (GEE) regression model to examine mammography appointment adherence in two analytical models. In the first model, we included all patients in the baseline and intervention period (intent to treat analysis). In the second model, we included only patients in the intervention period to examine those who did and did not complete the intervention (i.e., completed the reminder phone call). We modeled clustering across the 19 clinical sites using a logistic GEE regression (logit link with odds ratio) and an independent correlation structure. We analyzed age, season, wave, group, mammography mobile provider, the group variable and the five dichotomous variables for clinic racial/ethnic distribution, and for those in the intervention period, the $\mathrm{CHW}$ who made the appointment reminder call, if the patient answered the call, the number of reminder call attempts, and language independently in each of the models and added each additional variable as a covariate. The Quasi-Akaike information criterion (QIC) value was used to identity covariates to include in the final models. One-sided t-tests were conducted to analyze mean score changes (mean difference $<0$ ) between the clinic adoption and implementation survey responses. All analyses were performed using Stata 14.0 (College Station, TX) with $\alpha=0.05$ as the limit for statistical significance.

\section{Results}

Twenty-six FQHC and charity care clinical locations were approached for study enrollment (Fig. 1). Of the 26 clinics, 22 elected to adopt PMP (85\%). Two clinics enrolled (three clinical locations), but did not complete the trial yielding a total of 19 clinical sites for analysis. A total of 4408 patients were recruited for the study. Six patients were excluded from the final analysis because we were unable to determine mammography appointment adherence outcome from the Redcap responses. Of the 4402 in the final analysis, 2078 were enrolled in the baseline period and 2324 were enrolled in the intervention period (Table 1). Women aged 45 to 54 years old made up the largest age group in both periods (baseline-45\%; intervention-41\%). The patients in the baseline intervention had a higher percentage of mammography attendance in the Summer (baseline-47\%; intervention-18\%) compared to those intervention period who had a higher attendance in the Spring (baseline- $8 \%$;
Fig. 1 Flowchart showing enrollment, allocation, followup, and analysis of clinics and patients in the Peace of Mind Program (PMP) study trial. Description: Black and white graphic with a downward sequence of stages of enrollment, allocation, follow up, and analysis with indicated study participant sample size in each stage

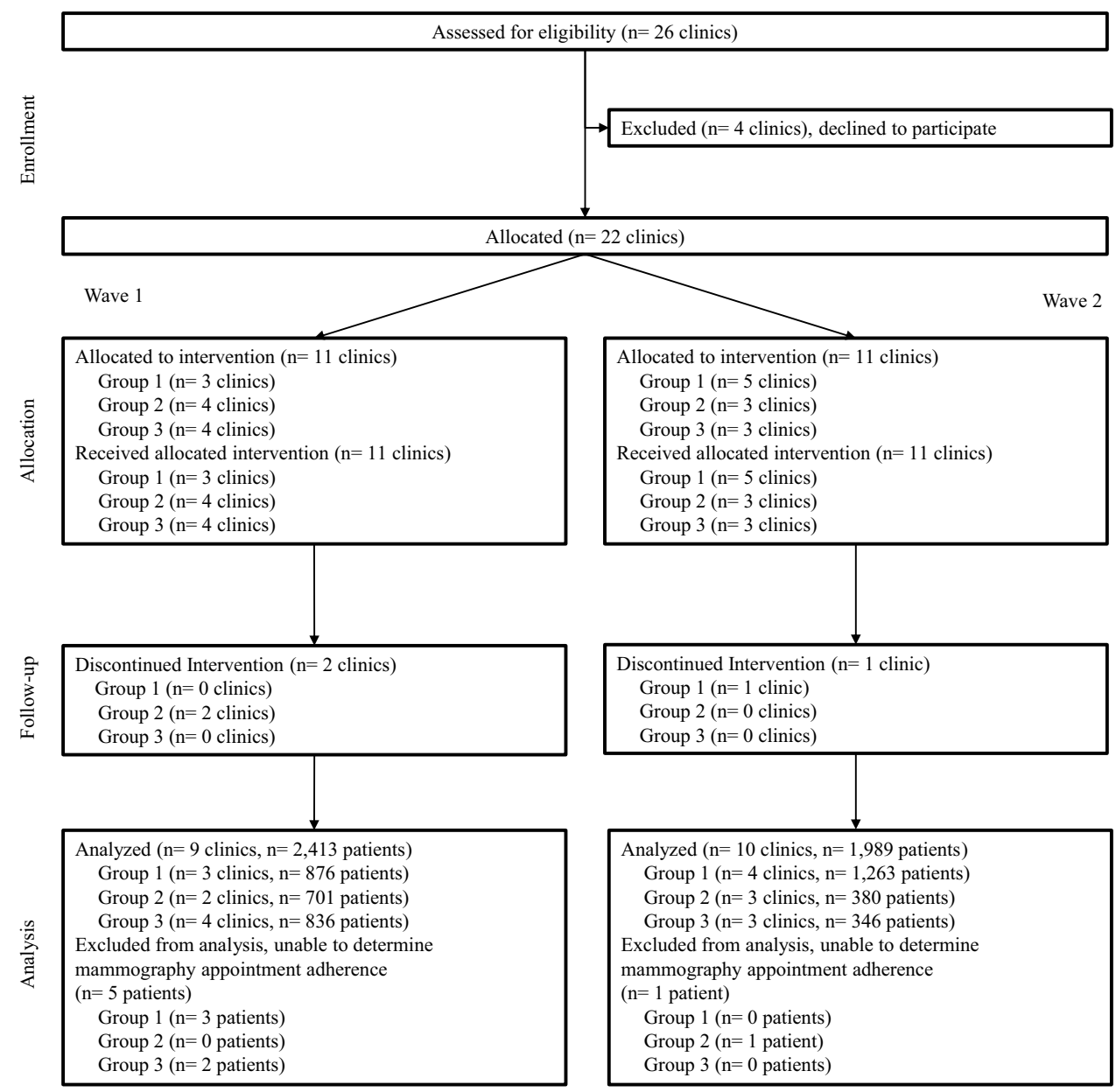


Table 1 Descriptive statistics of patient, intervention, clinic, and implementation variables

\begin{tabular}{|c|c|c|c|c|}
\hline \multirow[b]{2}{*}{ Variable } & \multicolumn{2}{|l|}{$\begin{array}{l}\text { All Patients } \\
(N=4402)\end{array}$} & \multicolumn{2}{|l|}{$\begin{array}{l}\text { Intervention Period } \\
(N=2324)\end{array}$} \\
\hline & $\begin{array}{l}\text { Baseline } \\
\text { Period } \\
(n=2078)\end{array}$ & $\begin{array}{l}\text { Intervention } \\
\text { Period } \\
(n=2324)\end{array}$ & $\begin{array}{l}\text { Did not complete } \\
\text { intervention } \\
(n=752)\end{array}$ & $\begin{array}{l}\text { Completed intervention } \\
(n=1572)\end{array}$ \\
\hline \multicolumn{5}{|l|}{ Patient Variables } \\
\hline \multicolumn{5}{|l|}{ Age category (years) } \\
\hline 25 to 44 & $519(25.0 \%)$ & $549(23.6 \%)$ & $174(23.1 \%)$ & $375(23.9 \%)$ \\
\hline 45 to 54 & $929(44.7 \%)$ & $958(41.2 \%)$ & $317(42.2 \%)$ & $641(40.8 \%)$ \\
\hline 55 and older & $630(30.3 \%)$ & $817(35.2 \%)$ & $261(34.7 \%)$ & $556(35.4 \%)$ \\
\hline \multicolumn{5}{|l|}{ Intervention Variables } \\
\hline \multicolumn{5}{|l|}{ Season (months) } \\
\hline Winter (Jan-March) & $382(18.4 \%)$ & $537(23.1 \%)$ & $186(24.7 \%)$ & $351(22.3 \%)$ \\
\hline Spring (Apr-June) & $172(8.3 \%)$ & $842(36.2 \%)$ & $256(34.0 \%)$ & $586(37.3 \%)$ \\
\hline Summer (July—Sep) & $981(47.2 \%)$ & $414(17.8 \%)$ & $145(19.3 \%)$ & $269(17.1 \%)$ \\
\hline Fall (Oct-Dec) & $543(26.1 \%)$ & $531(22.9 \%)$ & $165(22.0 \%)$ & $366(23.3 \%)$ \\
\hline \multicolumn{5}{|l|}{ Wave } \\
\hline 1 & $1233(59.3 \%)$ & $1180(50.8 \%)$ & $434(57.7 \%)$ & $746(47.5 \%)$ \\
\hline 2 & $845(40.7 \%)$ & $1144(49.2 \%)$ & $318(42.3 \%)$ & $826(52.5 \%)$ \\
\hline \multicolumn{5}{|l|}{ Group } \\
\hline 1 & $543(26.1 \%)$ & $1596(68.7 \%)$ & $484(64.4 \%)$ & $1112(70.7 \%)$ \\
\hline 2 & $676(32.5 \%)$ & $405(17.4 \%)$ & $169(22.5 \%)$ & $236(15.0 \%)$ \\
\hline 3 & $859(41.3 \%)$ & $323(13.9 \%)$ & $99(13.2 \%)$ & $224(14.3 \%)$ \\
\hline \multicolumn{5}{|l|}{ Clinic Variables } \\
\hline \multicolumn{5}{|l|}{ Mammography Mobile Provider } \\
\hline Provider 1 & $919(44.2 \%)$ & $627(27.0 \%)$ & $220(29.3 \%)$ & $407(25.9 \%$ \\
\hline Provider 2 & $995(47.9 \%)$ & $1218(52.4 \%)$ & $403(53.6 \%)$ & $815(51.8 \%)$ \\
\hline Provider 3 & $164(7.9 \%)$ & $479(20.6 \%)$ & $129(17.2 \%)$ & $350(22.3 \%)$ \\
\hline \multicolumn{5}{|l|}{ Clinic racial distribution $^{\mathrm{a}}$} \\
\hline Non-Hispanic Black & $168(8.1 \%)$ & $119(5.1 \%)$ & $42(5.6 \%)$ & $77(4.9 \%)$ \\
\hline Non-Hispanic white & $164(7.9 \%)$ & $415(17.9 \%)$ & $119(15.8 \%)$ & $296(18.8 \%)$ \\
\hline Non-Hispanic other & $108(5.2 \%)$ & $145(6.2 \%)$ & $9(1.2 \%)$ & $136(8.7 \%)$ \\
\hline Hispanic & $1591(76.6 \%)$ & $1627(70.0 \%)$ & $574(76.3 \%)$ & $1053(67.0 \%)$ \\
\hline Multi-racial/ethnicity group & $47(2.3 \%)$ & $18(0.77 \%)$ & $8(1.1 \%)$ & $10(0.64 \%)$ \\
\hline \multicolumn{5}{|l|}{ Implementation Variables } \\
\hline $\mathrm{CHW}$ calling patient & - & - & & \\
\hline Clinic staff & - & - & $242(32.2 \%)$ & $405(25.8 \%)$ \\
\hline BHCT staff & - & - & $510(67.8 \%)$ & $1167(74.2 \%)$ \\
\hline Answered reminder call & - & - & & \\
\hline No & - & - & $465(61.8 \%)$ & $0(0 \%)$ \\
\hline Yes & - & - & $287(38.2 \%)$ & $1572(100 \%)$ \\
\hline Number of reminder calls $(n=1852)^{\mathrm{b}}$ & - & - & & \\
\hline 1 call & - & - & $273(45.7 \%)$ & $908(72.35 \%)$ \\
\hline Multiple calls ( 2 or 3 calls) & - & - & $324(54.3 \%)$ & $347(27.65 \%)$ \\
\hline Language of reminder call $(n=1817)^{\mathrm{b}}$ & - & - & & \\
\hline English & - & - & $124(50.6 \%)$ & $608(39.0 \%)$ \\
\hline Spanish & - & - & $121(49.4 \%)$ & $828(53.0 \%)$ \\
\hline Vietnamese & - & - & $0(0 \%)$ & $125(8.0 \%)$ \\
\hline
\end{tabular}

${ }^{\mathrm{a}}$ Non-Hispanic Black, Non-Hispanic other, and Hispanic ${ }^{\mathrm{b}}$ Missing data 
intervention-36\%). Over half of patients in both periods were in wave 1 (baseline-59\%; intervention-51\%). Group 3 was largest in the baseline intervention (baseline-41\%; intervention-14\%) while group 1 was the largest in the intervention period (baseline-26\%; intervention-69\%). Mammography provider 2 who had no existing reminder call or group education was the largest mammography mobile provider to women in both periods (baseline- $48 \%$; intervention-52\%). Hispanic women were the largest served racial/ethnicity group across clinics in both periods (baseline-77\%; intervention-70\%).

Of the 2324 patients in the intervention period, 1572 completed the intervention. Patient age group, season, and mammography mobile provider were similar across those who did and did not complete the intervention (Table 1). The percentage of patients who did not complete the intervention was higher in wave 1 (did not complete-58\%; completed-47\%) while $71 \%$ of those who completed the intervention were in group 1. With Hispanic women being the largest racial/ethnicity group, a higher percentage of those who did not complete the intervention were from a clinic serving predominately Hispanic women (did not complete-76\%; completed-67\%). A higher percentage of those who completed the intervention received a call from a BHCTexas CHW compared to a clinic CHW (did not complete-68\%; completed-74\%). For those who did not complete the intervention, $38 \%$ answered the reminder call. For those who completed the intervention, $72 \%$ received one reminder phone call compared to $46 \%$ of those who did not complete the intervention. A higher percentage of patients who did not complete the intervention received a call in English (did not complete-51\%; completed-39\%) while a higher percentage of those who completed the intervention received the call in Spanish (did not complete-49\%; completed-53\%). All patients who received a call in Vietnamese completed the intervention.

\section{Mammography Appointment Adherence}

In the bivariate analysis, multiple statistically significant differences in mammography appointment adherence were identified (Table 2). A statistically significant difference in appointment adherence was observed between periods $(p<0.05)$, completion of the intervention $(p<0.001)$, wave $(p<0.01)$, group $(p<0.001)$, mammography mobile provider $(p<0.001)$, and clinic racial distribution $(p<0.001)$. A marginal trend toward significance $(p=0.058)$ was observed for both age and season. Those who attended or rescheduled their mammography appointment were more likely to be in the intervention period, complete the intervention, be in wave 1 and group 1, be served by a clinic serving predominately non-Hispanic women who identified with a race other than Black or white, and be served by mammography mobile provider 1 who had an existing reminder call and group education program compared to those who did not show or cancelled their appointment. The number of reminders call attempts, if the patient answered the reminder call, and language of the reminder call were statistically significant $(p<0.001)$. Those who attended or rescheduled their mammography appointment were more likely to answer the reminder call, receive one reminder call, and receive a reminder call in Spanish or Vietnamese compared to those who did not show or cancelled their appointment. Among the 2078 patients in the baseline period, 448 (22\% no-show rate) did not show up to their appointment, whereas among the 2324 patients in the intervention period 438 (19\% noshow rate) did not show up to their appointment. Among the 752 patients who did not complete intervention 205 (27\% no-show rate) did not show up, whereas among the 1572 patients who completed the intervention 233 (15\% no-show rate) did not show up. Table 3 includes the multivariable GEE logistic regression performed to fit the covariates to mammography appointment adherence by baseline and intervention period and completion of the intervention. In the first model, the intervention period, relative to the baseline period, was associated with higher odds of attending or rescheduling a mammography appointment $(\mathrm{OR}=1.30$; $p<0.01)$. The age group of 25 to 44 years was associated with lower odds compared to the 55 years and older age group $(\mathrm{OR}=0.73 ; p<0.001)$. Mammography mobile provider 2 was associated with lower odds $(\mathrm{OR}=0.60 ; p<0.01)$ compared to mammography mobile provider 1 . In the second model, completing the intervention, relative to not completing, was associated with higher odds of attending or rescheduling an appointment $(\mathrm{OR}=1.62 ; p<0.01)$. As in the first model, the age group of 25 to 44 years was associated with lower odds compared to the 55 years and older age group $(\mathrm{OR}=0.71 ; p<0.05)$. Relative to receiving one reminder call, receiving multiple reminder call attempts was also associated with lower odds attending or rescheduling an appointment $(\mathrm{OR}=0.78 ; p<0.05)$.

\section{Implementation}

A total of 20 clinics completed the adoption survey prior to implementation, with 15 of these clinics adopting and implementing the intervention. Of those 15 clinics who completed the adoption survey and implemented the intervention, eight completed the implementation survey at eight weeks post implementation. While we observed a statistically significant $(p<0.05)$ decreases in Inner Setting overall and in CultureEffort and Implementation Climate (Inner Setting constructs), potential directional trends can be identified. We observed a decrease in intervention Characteristic constructs such as Relative Advantage, Trialability, and Compatibility, but an increase in Complexity (i.e., easier to implement). We observed an 
Table 2 Association of patient, intervention, clinic, and implementation variables with mammography appointment adherence $(N=4402)$

\begin{tabular}{|c|c|c|c|}
\hline Variable & $\begin{array}{l}\text { No Show or Cancelled } \\
(N=886)\end{array}$ & $\begin{array}{l}\text { Attended or Rescheduled } \\
(N=3516)\end{array}$ & $p$ value \\
\hline Study period & & & $<0.05$ \\
\hline Baseline period & $448(50.6 \%)$ & $1630(46.5 \%)$ & \\
\hline Intervention period & $438(49.4 \%)$ & $1886(53.6 \%)$ & \\
\hline Intervention period $(\mathrm{n}=2324)$ & & & $<0.001$ \\
\hline Did not complete intervention & $205(46.8 \%)$ & $547(29.0 \%)$ & \\
\hline Completed intervention & $233(53.2 \%)$ & $1339(71.0 \%)$ & \\
\hline \multicolumn{4}{|l|}{ Patient Variables } \\
\hline Age category (years) & & & NS \\
\hline 25 to 44 & $239(27.0 \%)$ & $829(23.6 \%)$ & \\
\hline 45 to 54 & $379(42.8 \%)$ & $1508(42.9 \%)$ & \\
\hline 55 and older & $268(30.3 \%)$ & $1179(33.5 \%)$ & \\
\hline \multicolumn{4}{|l|}{ Intervention Variables } \\
\hline Season (months) & & & NS \\
\hline Winter (Jan—March) & $178(20.1 \%)$ & $741(21.1 \%)$ & \\
\hline Spring (Apr-June) & $202(22.8 \%)$ & $812(23.1 \%)$ & \\
\hline Summer (July—Sep) & $299(33.8 \%)$ & $1096(31.2 \%)$ & \\
\hline Fall (Oct-Dec) & $207(23.4 \%)$ & $867(24.7 \%)$ & \\
\hline Wave & & & $<0.01$ \\
\hline 1 & $450(50.8 \%)$ & $1963(55.8 \%)$ & \\
\hline 2 & $436(49.2 \%)$ & $1553(44.2 \%)$ & \\
\hline Group & & & $<0.001$ \\
\hline 1 & $396(44.7 \%)$ & $1743(49.6 \%)$ & \\
\hline 2 & $201(22.7 \%)$ & $880(25.0 \%)$ & \\
\hline 3 & $289(32.6 \%)$ & $893(25.4 \%)$ & \\
\hline \multicolumn{4}{|l|}{ Clinic Variables } \\
\hline \multicolumn{4}{|l|}{ Mammography Mobile Provider } \\
\hline Provider 1 & $252(28.4 \%)$ & $1294(36.8 \%)$ & $<0.001$ \\
\hline Provider 2 & $511(57.7 \%)$ & $1702(48.4 \%)$ & \\
\hline Provider 3 & $123(13.9 \%)$ & $520(14.8 \%)$ & \\
\hline Clinic racial distribution & & & $<0.001$ \\
\hline Non-Hispanic Black & $97(11.0 \%)$ & $190(5.4 \%)$ & \\
\hline Non-Hispanic white & $121(13.7 \%)$ & $480(13.0 \%)$ & \\
\hline Non-Hispanic other & $12(1.4 \%)$ & $241(6.9 \%)$ & \\
\hline Hispanic & $650(73.4 \%)$ & $2568(73.0 \%)$ & \\
\hline Multi-racial/ethnicity $^{a}$ & $6(0.68 \%)$ & $59(1.7 \%)$ & \\
\hline \multicolumn{4}{|l|}{ Implementation Variables } \\
\hline CHW calling patient $(n=2324)$ & & & NS \\
\hline Clinic staff & $114(26.0 \%)$ & $533(28.3 \%)$ & \\
\hline BHCT staff & $324(74.0 \%)$ & $1353(71.7 \%)$ & \\
\hline Answered reminder call $(n=2324)$ & & & $<0.001$ \\
\hline No & $139(31.7 \%)$ & $326(17.3 \%)$ & \\
\hline Yes & $299(68.3 \%)$ & $1560(82.7 \%)$ & \\
\hline Number of reminder calls $(n=1852)^{\mathrm{b}}$ & & & $<0.001$ \\
\hline 1 call & $202(54.0 \%)$ & $979(66.2 \%)$ & \\
\hline Multiple calls ( 2 or 3 calls) & $172(46.0 \%)$ & $499(33.8 \%)$ & \\
\hline Language of reminder call $(n=1806)^{\mathrm{b}}$ & & & $<0.001$ \\
\hline English & $148(52.5 \%)$ & $584(38.3 \%)$ & \\
\hline Spanish & $132(46.8 \%)$ & $817(53.6 \%)$ & \\
\hline Vietnamese & $2(0.71 \%)$ & $123(8.1 \%)$ & \\
\hline
\end{tabular}

${ }^{a}$ Non-Hispanic Black, Non-Hispanic other, and Hispanic ${ }^{\mathrm{b}}$ Missing data 
Table 3 Generalized estimating equation (GEE) models assessing mammography appointment adherence by baseline and intervention period and completion of the intervention

\begin{tabular}{|c|c|c|c|c|}
\hline \multirow[b]{2}{*}{ Variable } & \multicolumn{2}{|l|}{$\begin{array}{l}\text { Model } 1 \\
(N=4402)\end{array}$} & \multicolumn{2}{|l|}{$\begin{array}{l}\text { Model } 2 \\
(N=1852)\end{array}$} \\
\hline & Odds Ratio & $S E$ & Odds Ratio & $S E$ \\
\hline Study period & $1.30 * *$ & 0.12 & - & - \\
\hline Intervention period $^{\mathrm{a}}$ & - & - & $1.62 * *$ & 0.30 \\
\hline Age category (years) & - & - & & \\
\hline 25 to 44 vs 55 and older & $0.73 * * *$ & 0.06 & $0.71 *$ & 0.12 \\
\hline 45 to 54 vs 55 and older & 0.87 & 0.09 & 1.04 & 0.19 \\
\hline \multicolumn{5}{|l|}{ Season (months) } \\
\hline Spring vs Winter & 0.95 & 0.13 & 0.98 & 0.22 \\
\hline Summer vs Winter & 0.95 & 0.14 & 0.84 & 0.25 \\
\hline Fall vs Winter & 1.03 & 0.16 & 1.16 & 0.33 \\
\hline Wave & 0.95 & 0.25 & 0.98 & 0.28 \\
\hline $\begin{array}{l}\text { Mobile Mammography } \\
\text { Provider } 2\end{array}$ & $0.60 * *$ & 0.11 & 0.77 & 0.16 \\
\hline $\begin{array}{l}\text { Mobile Mammography } \\
\text { Provider } 3\end{array}$ & 0.63 & 0.2 & 1.09 & 0.35 \\
\hline $\begin{array}{l}\text { Clinic racial distribution- } \\
\text { Hispanic }\end{array}$ & 0.82 & 0.37 & 1.52 & 0.58 \\
\hline CHW calling patient & 0.96 & 0.12 & 0.87 & 0.12 \\
\hline Answered reminder call & - & - & 1.41 & 0.32 \\
\hline Number of reminder calls & - & - & $0.78 *$ & 0.09 \\
\hline
\end{tabular}

${ }^{\text {a }}$ Did not or did complete the intervention

$* p<.05 * * p<.01 * * * p<.001$

increase in the Inner Setting construct of Culture-Stress (i.e., improvements in staff stress and frustration) and in the Outer Setting constructs of Policies and Incentives and Patients Needs and Resources. The implementation survey included questions to assess motivation to participate, PMP enrollment, and influence of the adoption webinar. Across the eight clinics, a total of 16 clinic staff members completed these questions. Clinics staff members reported their motivation to participate in PMP included participating in an EBI, helping patients to understand the importance of mammography screening, and reducing mammography no-show rates. Staff members reported being more motivated to participate in PMP because of the partnership between BHCTexas and the researchers, compared to just their membership in BHCTexas. All but one clinic staff member who participated in the webinar reported the webinar influenced their decision to enroll in PMP. All clinic staff members found the enrollment for PMP easy.

\section{Discussion}

Within the cancer realm, a wide gulf between research and practice continues to lead to suboptimal EBI implementation [23]. We sought specifically to address these gaps in the development of PMP and in partnering with BHCTexas in this study $[15,16]$. We hypothesized that a communityacademic partnership would positively impact adoption and implementation. Bridging factors in implementation science consider relational ties, strength, processes and formal arrangements that connect the Inner and Outer Setting [24]. Recent research indicates a gap in the implementation science literature related to bridging and its impact on EBI implementation [24]. Using a community-academic partnership, focusing on internal and external incentives, addressing funding gaps, and staging implementation were all applied in the implementation strategies $[15,16]$. The implementation survey indicated this partnership motivated clinics to adopt. We found a directional (though not significant) increases in Outer setting constructs related to bridging (Patient Needs and Resources) and in Inner Setting constructs related to ease of implementation (Culture-Stress). We also found statistically significant decreases in some Inner Setting constructs (Culture-Effort and Implementation Climate). It is possible that 8 weeks post-implementation was too soon for staff to feel that sufficient systems to support PMP were in place in their clinics and that they had sufficient self-efficacy to lead without support. It also is possible that Inner Setting scores decreased at implementation due to staff gaining a more realistic understanding of what successful EBI implementation takes once being exposed to PMP in daily operations. This change could reflect the dynamic nature of working in under-resourced, high stress clinical environments. Our previous work in PMP development and results from this study provide an opportunity to expand the knowledge base related to bridging strategies on EBI implementation, highlight practical approaches that can be replicated or built upon by other implementation scientists and identifies opportunities where further study is warranted (such as the effect on Inner Setting constructs). No differences in attendance were observed by race in the intervention period model indicating that PMP addressed barriers across diverse population groups. Further, language in which PMP was delivered, which was statistically significant in bivariate analysis, was not significant in the intervention period model. This indicates that PMP delivery was effective in multiple languages. Marginal trends observed for season disappeared in the intervention period model indicating PMP successfully addressed structural barriers that have been shown to affect patient appointment attendance during the year (such as lack of time off). Effect of mobile provider's usual care reminder and education practices on attendance also disappeared in the GEE model for women who completed our program, indicating that PMP successfully improved appointment adherence to an equivalent level across providers despite this variation in routine care delivery.

Limitations of the study include not randomizing the start dates of clinics, which could have resulted in bias. 
We evaluated the effect of wave and group in our GEE models and found no impact. Women of younger age did retain lower odds of attendance even when completing PMP, indicating that there may be unique barriers in this age group. This finding should be investigated further to understand if younger women face unique barriers to adherence. We also conducted the implementation survey at eight weeks post implementation for each site. There is no literature to indicate the ideal timing for assessment of these constructs after implementation. The adoption and implementation survey were provided to all clinic staff, though not all sites completed them despite reminders. Due to low numbers of staff completing both surveys, we were not able to perform more advanced statistical analysis. Due to the low response rate, the external generalizability of the findings should be considered, as the staff or clinics completing the survey could have had differences in motivation or readiness to implement compared to those who did not complete the survey. High rates of staff turnover did occur in the clinics during the study which required ongoing training and support and may impact sustainability of the implementation strategies (e.g., trainings, stakeholder meetings, support) and the intervention.

\section{Conclusion}

The findings address a research to practice gap in understanding effective implementation of a mammography EBI. Identifying clinic readiness for implementation and providing implementation strategies to support clinics are important to promote the successful uptake of an EBI in safety net clinics.

Supplementary Information The online version contains supplementary material available at https://doi.org/10.1007/s13187-021-02116-w.

Acknowledgements The authors would like to acknowledge the Breast Health Collaborative of Texas (BHCTexas) and participating clinical partners for their ongoing collaboration. The authors would like to acknowledge the project funder, the Agency for Healthcare Research and Quality (AHRQ), for their research support.

Author Contribution Suja S. Rajan, Gretchen H. Walton, and Linda Highfield conceptualized the study. Jennifer Holcomb led manuscript development and prepared the final manuscript. Jennifer Holcomb, Suja S. Rajan, and Jiali Sun conducted the data analysis. Jennifer Holcomb, Gayla M. Ferguson, Gretchen H. Walton, and Linda Highfield were contributors to the manuscript writing and revisions. All authors have read and approved the manuscript.

Funding Funding for this study is provided by the Agency for Healthcare Research and Quality (AHRQ) (Grant number: 1R18HS023255-01).
Data Availability The datasets generated and/or analyzed during the current study are not publicly available due identifying clinic and patient information but are available from the corresponding author on reasonable request.

Code Availability Not applicable.

\section{Declarations}

Ethics Approval and Consent to participate The study received IRB approval from the Committee for the Protection of Human Subjects at The University of Texas Health Science Center at Houston (UTHealth).

Consent for Publication Not applicable.

Conflict of Interest The authors declare no competing interests.

Open Access This article is licensed under a Creative Commons Attribution 4.0 International License, which permits use, sharing, adaptation, distribution and reproduction in any medium or format, as long as you give appropriate credit to the original author(s) and the source, provide a link to the Creative Commons licence, and indicate if changes were made. The images or other third party material in this article are included in the article's Creative Commons licence, unless indicated otherwise in a credit line to the material. If material is not included in the article's Creative Commons licence and your intended use is not permitted by statutory regulation or exceeds the permitted use, you will need to obtain permission directly from the copyright holder. To view a copy of this licence, visit http://creativecommons.org/licenses/by/4.0/.

\section{References}

1. Howlader N, Noone AM, Krapcho M et al (eds) (2020) SEER Cancer Statistics Review, 1975-2018, National Cancer Institute. Bethesda, MD, https://seer.cancer.gov/csr/1975_2018/, Accessed 19 Oct 2021

2. American Cancer Society (2021) American Cancer Society Recommendations for the Early Detection of Breast Cancer. https:// www.cancer.org/cancer/breast-cancer/screening-tests-and-earlydetection/american-cancer-society-recommendations-for-theearly-detection-of-breast-cancer.html Accessed 30 Apr 2021

3. Rajan SS, Begley CE, Kim B (2014) Breast cancer stage at diagnosis among medically underserved women screened through the Texas breast and cervical cancer services. Popul Health Manag 17(4):202-210. https://doi.org/10.1089/pop.2013.0079

4. Irvin VL, Zhang Z, Simon MS et al (2020) Comparison of mortality among participants of women's health initiative trials with screening-detected breast cancers vs interval breast cancers. JAMA Netw Open 3(6):e207227. https://doi.org/10.1001/jaman etworkopen.2020.7227

5. Duffy SW, Tabár L, Yen AM et al (2021) Beneficial effect of consecutive screening mammography examinations on mortality from breast cancer: a prospective study. Radiology 299(3):541-547. https://doi.org/10.1148/radiol.2021203935

6. Rajan SS, Begley CE, Highfield LD, Kim B (2015) Survival benefits of treatment access among underserved breast cancer patients diagnosed through the Texas breast and cervical cancer services program. J Public Health Manag Pract 21(5):477-486. https://doi. org/10.1097/PHH.0000000000000255

7. Thompson B, Hohl SD, Molina Y et al (2018) Breast cancer disparities among women in underserved communities in the USA. 
Curr Breast Cancer Rep 10(3):131-141. https://doi.org/10.1007/ s12609-018-0277-8

8. Gathirua-Mwangi W, Cohee A, Tarver WL et al (2018) Factors associated with adherence to mammography screening among insured women differ by income levels. Womens Health Issues 28(5):462-469. https://doi.org/10.1016/j.whi.2018.06.001

9. Vang S, Margolies LR, Jandorf L (2018) Mobile mammography participation among medically underserved women: a systematic review. Prev Chronic Dis 15:180291. https://doi.org/10.5888/ pcd15.180291

10. Henderson V, Tossas-Milligan K, Martinez E et al (2020) Implementation of an integrated framework for a breast cancer screening and navigation program for women from underresourced communities. Cancer 126:2481-2493. https://doi.org/10.1002/ cncr.32843

11. Sheppard VB, Wang JH, Eng-Wong J, Martin SH, Hurtado-deMendoza A, Luta G (2013) Promoting mammography adherence in underserved women: The telephone coaching adherence study. Contemp Clin Trials 35(1):35-42. https://doi.org/10.1016/j.cct. 2013.02.005

12. Nonzee NJ, Ragas DM, Luu TH et al (2015) Delays in cancer care among low-income minorities despite access. J Womens Health 24(6):506-514. https://doi.org/10.1089/jwh.2014.4998

13. Allen CL, Harris JR, Hannon PA et al (2014) Opportunities for improving cancer prevention at federally qualified health centers. J Cancer Educ 29(1):30-37. https://doi.org/10.1007/ s13187-013-0535-4

14. Cialdella-Kam L, Sabado P, Bispeck MK et al (2012) Implementing cancer prevention into clinical practice. J Cancer Educ 27(2):136-143. https://doi.org/10.1007/s13187-012-0331-6

15. Highfield L, Rajan SS, Valerio MA, Walton G, Fernandez ME, Bartholomew LK (2015) A non-randomized controlled stepped wedge trial to evaluate the effectiveness of a multi-level mammography intervention in improving appointment adherence in underserved women. Implement Sci 10(1):1-8. https://doi.org/10. 1186/s13012-015-0334-x

16. Highfield L, Valerio MA, Fernandez ME, Eldridge-Bartholomew LK (2018) Development of an implementation intervention using intervention mapping to increase mammography among low income women. Front Public Health 6:300. https://doi.org/10. 3389/fpubh.2018.00300

17. Holcomb J, Ferguson GM, Sun J, Walton GH, Highfield L (2021) Stakeholder engagement in adoption, implementation, and sustainment of an evidence-based intervention to increase mammography adherence among low-income women. J Cancer Educ. https://doi.org/10.1007/s13187-021-01988-2

18. Onitilo AA, Liang H, Stankowski RV et al (2014) Geographical and seasonal barriers to mammography services and breast cancer stage at diagnosis. Rural Remote Health 14(3):180

19. Tsuruda KM, Bhargava S, Mangerud G, Sagstad S, Hofvind SS (2017) Monthly variation in mammographic screening attendance in Norway. Eur J Public Health 27(6):1095-1097. https://doi.org/ 10.1093/eurpub/ckx137

20. Fernandez ME, Liang S, Jacobs SR, Taplin SH, Weiner BJ (2015) Pressing ahead: developing and testing of new measures in implementation science. Implementation Science. BioMed Central 10(1):1-3. https://doi.org/10.1186/1748-5908-10-S1-A14

21. Fernández ME, Melvin CL, Leeman J et al (2014) The cancer prevention and control research network: an interactive systems approach to advancing cancer control implementation research and practice. Cancer Epidemiol Prev Biomark 23(11):2512-2521. https://doi.org/10.1158/1055-9965.EPI-14-0097

22. Kegler MC, Liang S, Weiner BJ et al (2018) Measuring constructs of the consolidated framework for implementation research in the context of increasing colorectal cancer screening in federally qualified health center. Health Serv Res 53(6):4178-4203. https:// doi.org/10.1111/1475-6773.13035

23. Rositch AF, Unger-Saldaña K, DeBoer RJ, Ng'ang'a A, Weiner BJ (2020) The role of dissemination and implementation science in global breast cancer control programs: frameworks, methods, and examples. Cancer 126:2394-2404. https://doi.org/10.1002/ cncr.32877

24. Lengnick-Hall R, Stadnick NA, Dickson KS, Moullin JC, Aarons GA (2021) Forms and functions of bridging factors: specifying the dynamic links between outer and inner contexts during implementation and sustainment. Implement Sci 16(1):1-3. https://doi. org/10.1186/s13012-021-01099-y

Publisher's Note Springer Nature remains neutral with regard to jurisdictional claims in published maps and institutional affiliations. 